\title{
SOME CONSTRUCTIONS OF RINGS OF FINITE GLOBAL DIMENSION
}

\author{
by J. C. ROBSON
}

(Received 20 June, 1983)

In the study of idealizer rings in [14], the global dimensions of certain subrings were calculated. This work was extended by Goodearl [9]. Here it is shown, in Section 1, that the techniques involved can be used rather more generally than was apparent there. The results concern a ring $S$ with a right ideal $A$ such that $S A=S$, and a subring $R$ of $S$ containing $A$. They describe $g l \operatorname{dim} R$ in terms of $g l \operatorname{dim} S$ and the projective dimensions of simple subfactors of $S / R$ or $R / A$, provided that these $R$-modules are sufficiently well conditioned.

This is applied in Section 2, to construct examples of Noetherian semiprime rings of finite global dimension which are not direct sums of prime rings. These examples, which provide yet another contrast with the commutative theory, are of interest in the light of results of Brown, Hajarnavis and MacEacharn $[1,2,3]$. In fact, this paper, to some extent, is a response to a question by Hajarnavis in this context.

In Section 3, the technique is used to calculate global dimensions of generalizations of certain 'tiled' rings of the form studied by Fields [7], Tarsy $[17,18]$ and V. A. Jategaonkar $[10,11,12]$, and a variant used by Stafford and Warfield [16].

Some of the results in this paper were obtained during an SERC supported Ring Theory Symposium at the University of Warwick. This support, and the hospitality of the Mathematics Institute, are gratefully acknowledged.

1. Basic Techniques. Throughout this section, let $S$ be a ring (with 1), $A$ a right ideal of $S$ such that $S A=S$ and $R$ a proper subring of $S$ containing $A$. The results below aim to measure the right global dimension of $R$ in terms of $\operatorname{rtgl} \operatorname{dim} S$ and the behaviour of $S / A$ as an $R$-module.

Note that $R A$ is a two-sided ideal of $R$, and is also a right ideal of $S$. Moreover, $S(R A)=S$. Thus, in effect, we can always choose $A$ to be an ideal of $R$-and then $R$ is a subidealizer of $A$ in $S$. However, these considerations are not required for what follows.

LEMMA 1.1. (i) If $M$ is any right $S$-module then p.d. $M_{R}=$ p.d. $M_{S}$.

(ii) $\mathrm{rt} \mathrm{gl} \operatorname{dim} R \geqslant \sup \{\mathrm{rt} \mathrm{gl} \operatorname{dim} S, 1\}$.

(iii) p.d. $(R / A)_{R}=$ p.d. $(S / A)_{S}$.

Proof. (i) See [14, Lemma 2.8].

(ii) From (i), $\mathrm{rt} g l \operatorname{dim} R \geqslant \mathrm{rt} g l \operatorname{dim} S$. Suppose that $\mathrm{rtgl} \operatorname{dim} R=0$, and so $R$ is semisimple. Now $R A$ is an ideal of $R$, with $\mathrm{rt}$ ann $R A=0$ since $S A=S$. Therefore $R=R A=R A S=R S=S$, a contradiction.

(iii) It is easy to check that $A_{R}$ has an idempotent generator if and only if $A_{S}$ has. In other words, the short exact sequence of right $R$-modules, $0 \rightarrow A \rightarrow R \rightarrow R / A \rightarrow 0$, splits

Glasgow Math. J. 26 (1985) 1-12. 
if and only if the sequence of right $S$-modules, $0 \rightarrow A \rightarrow S \rightarrow S / A \rightarrow 0$, splits. By (i), p.d. $A_{R}=$ p.d. $A_{S}$, and so p.d. $(R / A)_{R}=$ p.d. $(S / A)_{S}$.

THEOREM 1.2. Suppose that $(S / R)_{R}$ has a finite series given by $S=B_{0} \supset B_{1} \supset \ldots \supset B_{k}=$ $R$ such that for each $i, B_{i} / B_{i+1}$ is a semisimple $R$-module. Then

$$
\mathrm{rt} \operatorname{gl} \operatorname{dim} R=\sup \{\mathrm{rt} \mathrm{gl} \operatorname{dim} S, 1, m\}
$$

where $m=\sup \{$ p.d. $U \mid U$ any simple subfactor module of $S / R\}$.

Proof. (Following [14, Proposition 2.11]). Let $\mathrm{rt} \operatorname{gl} \operatorname{dim} S=\boldsymbol{n}$. It is clear from Lemma 1.1(ii) that $\mathrm{rt} \operatorname{gl} \operatorname{dim} R \geqslant \sup \{n, 1, m\}$.

Next, let $D \triangleleft R_{R}$; then $D S \triangleleft S_{S}$ and $D S / D$ is a homomorphic image of a direct sum $(S / R)^{(I)}$ for some index set $I$. Let $\alpha:(S / R)^{(I)} \rightarrow D S / D$ be the epimorphism, and let $D_{i} / D=\alpha\left(\left(B_{i} / R\right)^{(I)}\right)$ with $D_{i} \supseteq D$ a submodule of $S_{R}$. Thus

$$
D S=D_{0} \supseteq D_{1} \supseteq \ldots \supseteq D_{k}=D .
$$

We make the induction hypothesis that p.d. $D_{i} \leqslant \sup \{n-1,0, m-1\}$ and will verify the corresponding result for $D_{i+1}$. Note, however, that the result is true for $D_{0}$, by Lemma 1.1(i).

Since $B_{i} / B_{i+1}$ is semisimple, so too is $D_{i} / D_{i+1}$. Hence p.d. $D_{i} / D_{i+1} \leqslant m$. This, together with the induction hypothesis, applied to the short exact sequence

$$
0 \rightarrow D_{i+1} \rightarrow D_{i} \rightarrow D_{i} / D_{i+1} \rightarrow 0
$$

shows that p.d. $D_{i+1} \leqslant \sup \{n-1,0, m-1\}$ as required. The result follows immediately.

The next, less precise, result relies on knowledge of $(R / A)_{R}$.

THEOREM 1.3. Suppose that $R / A$ has a finite series given by

$$
R=B_{0} \supset B_{1} \supset \ldots \supset B_{k}=A
$$

with each factor $B_{i} / B_{i+1}$ being a semisimple right $R$-module. Then

$$
\sup \{\mathrm{rt} \operatorname{gl} \operatorname{dim} S, m\} \leqslant \mathrm{rt} \mathrm{gl} \operatorname{dim} R \leqslant \sup \{\mathrm{rt} \operatorname{gl} \operatorname{dim} S, m+1\}
$$

where $m=\sup \{p . d . U \mid U$ a simple subfactor module of $R / A\}$.

Proof. Let $D \triangleleft R_{R}$; so $D A \triangleleft S_{S}$ and $D A \subseteq D$. There exists an epimorphism $\alpha:(R / A)^{(I)} \rightarrow D / D A$ for some index set $I$. Let $D_{i} \supseteq D A$ be such that $D_{i} / D A=$ $\alpha\left(\left(B_{i} / A\right)^{(I)}\right)$. This gives the series

$$
D=D_{0} \supset D_{1} \supset \ldots \supset D_{k}=D A .
$$

We make the induction hypothesis that p.d. $D_{i+1} \leqslant \sup \{n-1, m\}$ where $n=\mathrm{rt} \operatorname{gl} \operatorname{dim} S$. Now p.d. $D_{k}=$ p.d. $D A_{s} \leqslant \sup (n-1,0)$, using Lemma 1.1(i). So the induction is well based. In the short exact sequence

$$
0 \rightarrow D_{i+1} \rightarrow D_{i} \rightarrow D_{i} / D_{i+1} \rightarrow 0
$$


p.d. $D_{i+1} \leqslant \sup \{n-1, m\}$ and p.d. $D_{i} / D_{i+1} \leqslant m$. Hence, p.d. $D_{i} \leqslant \sup \{n-1, m\}$. This applies to $D$ and shows that $\mathrm{rt} \operatorname{gl} \operatorname{dim} R \leqslant \sup \{n, m+1\}$.

Corollary $1.4\left[9\right.$, Theorem 2.2]. Suppose that $(R / A)_{R}$ is semisimple. Then

$\mathrm{rt} \mathrm{gl} \operatorname{dim} S \leqslant \mathrm{rt} \mathrm{gl} \operatorname{dim} R \leqslant \mathrm{rt} \operatorname{gl} \operatorname{dim} S+1$.

Proof. In this case $m=$ p.d. $(R / A)_{R}$. Therefore by Lemma $1.1($ iii $), m=$ p.d. $(S / A)_{S} \leqslant$ $\mathrm{rt} g \mathrm{gl} \operatorname{dim} S$. The corollary now follows.

The next theorem, which is a small extension of [14, Proposition 2.3 and 2.6], deals with chain conditions in this setting.

THEOREM 1.5. Suppose that $S$ is right Noetherian with $\mathrm{K} \operatorname{dim} S=\alpha$, and that $(S / A)_{R}$ is Noetherian with $\mathrm{K} \operatorname{dim}(S / A)_{R}=\beta$. Then $R$ is right Noetherian with $\sup \{\alpha, \beta\} \leqslant \mathrm{K} \operatorname{dim} R \leqslant$ $\beta+\alpha$.

Proof. [14, Proposition 2.3] shows that $R$ is right Noetherian. Moreover, since $S_{\mathrm{R}}$ is finitely generated, it is clear that $\mathrm{K} \operatorname{dim} R_{R}=\mathrm{K} \operatorname{dim} S_{R} \geqslant \mathrm{~K} \operatorname{dim} S_{\mathrm{S}}=\alpha$; and it is obvious that $\mathrm{K} \operatorname{dim} R \geqslant \beta$.

For the other inequality, we need to show, for any finitely generated right $S$-module $N$, that $\mathrm{K} \operatorname{dim} N_{\mathrm{R}} \leqslant \beta+\mathrm{K} \operatorname{dim} N_{\mathrm{S}}$. This we demonstrate by induction on $\mathrm{K} \operatorname{dim} N_{\mathrm{S}}=\gamma$ say. If $\gamma=0$, then $N_{S}$ has a finite composition series each factor of which has Krull dimension (over $R$ ) less than or equal to $\beta$, by [14, Proposition 1.4(a)(ii)]; and so $\mathrm{K} \operatorname{dim} N_{R} \leqslant \beta=$ $\beta+\gamma$. If $\gamma>0$, consider any descending chain $\left\{N_{k}\right\}$ of submodules of $N_{R}$. There are corresponding chains $\left\{N_{k} S\right\}$ and $\left\{N_{k} A\right\}$ of submodules of $N_{S}$; and so, for large $k$, $\mathrm{K} \operatorname{dim}\left(N_{k} S / N_{k+1} S\right)_{S}<\gamma$ and $\mathrm{K} \operatorname{dim}\left(N_{k} A / N_{k+1} A\right)_{S}<\gamma$. By the induction hypothesis, for each of these modules, $\mathrm{K} \mathrm{dim} \operatorname{dim}_{R}<\beta+\gamma$. The lattice in Fig. 1 makes it clear that $\mathrm{K} \operatorname{dim} N_{k} / N_{k+1}<\beta+\gamma$, since $\mathrm{K} \operatorname{dim} N_{i} S / N_{i} A \leqslant \beta$ and $\gamma>0$. Hence $\mathrm{K} \operatorname{dim} N_{R} \leqslant \beta+\gamma$.

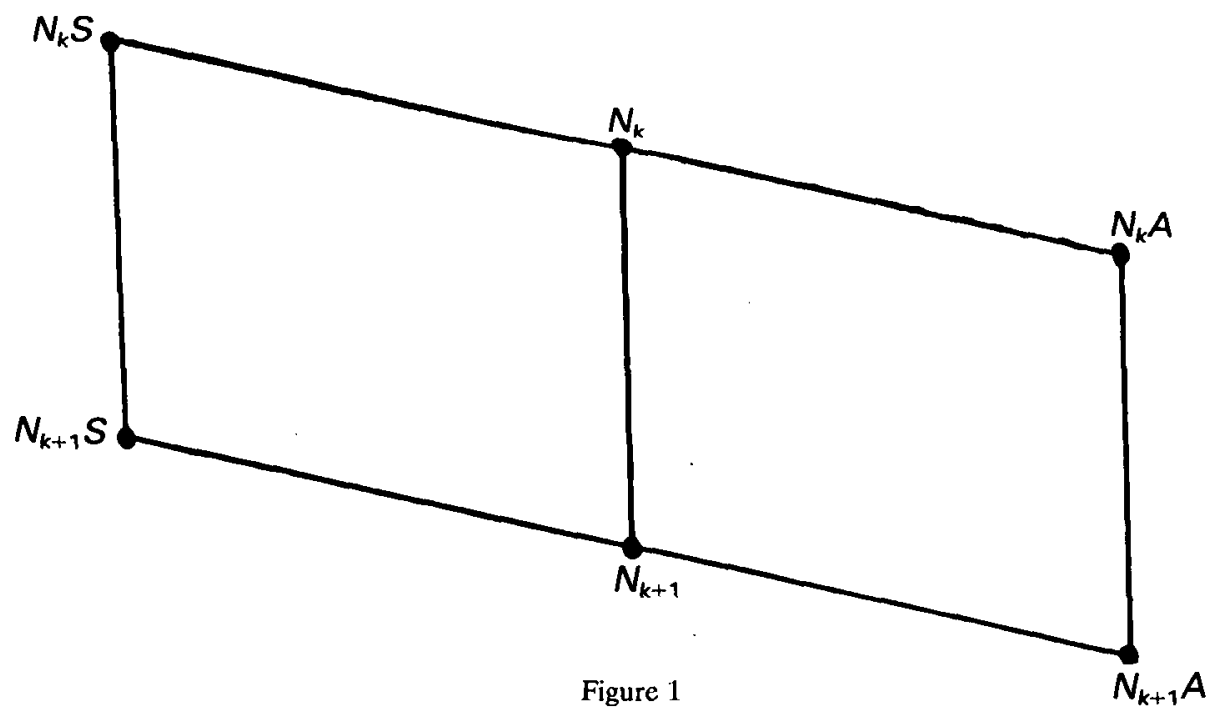


2. Semiprime rings. In this section we first describe a technique for constructing semiprime rings which are not direct sums of prime rings. Then, using the results of Section 1, we show that these can be arranged to be Noetherian and of finite global dimension.

Notation 2.1. Let $n$ be an integer, $n \geqslant 2$, and let $R_{i}$ be a ring, $A_{i}$ an ideal of $R_{i}$ for $i=1, \ldots, n$. Suppose that, for each $i$, there exists an isomorphism $\theta_{i}: R_{i} / A_{i} \rightarrow K$ fór some ring $K$. Then let $T$ be the subset of $\prod R_{i}$ defined by

$$
T=\left\{\left(r_{1}, \ldots, r_{n}\right) \mid \theta_{j}\left(r_{j}\right)=\theta_{i}\left(r_{i}\right) \text { for all } i, j\right\} .
$$

LEMMA 2.2. (i) $T$ is a ring;

(ii) $A_{i}$ embeds as an ideal of $T$ for each $i$ via the map $a_{i} \rightarrow\left(0, \ldots, 0, a_{i}, 0, \ldots, 0\right)$;

(iii) $T / \prod_{i=1}^{n} A_{i} \simeq K$;

(iv) $T / \prod_{\substack{i=1 \\ i \neq j}}^{n} A_{i} \simeq R_{j}$.

Proof. These are all easily verified.

LEMma 2.3. (i) If each $R_{i}$ is an indecomposable ring, then so too is $T$.

(ii) If each $R_{i}$ is right Noetherian with $\mathrm{K} \operatorname{dim} R_{i}=\alpha_{i}$, then $T$ is right Noetherian and $\mathrm{K} \operatorname{dim} T=\sup \left\{\alpha_{i}\right\}$.

(iii) If each $R_{\mathrm{i}}$ is prime then $T$ is semiprime but not prime.

Proof. (i) Suppose $T=e_{1} T \oplus e_{2} T$ is a decomposition of $T$ with $e_{1}, e_{2}$ being central orthogonal idempotents, and $1=e_{1}+e_{2}$. By Lemma 2.2(iv), each $e_{i}$ centralizes each $R_{\mathrm{i}}$. Thus $e_{1}, e_{2}$ are also central orthogonal idempotents of the ring $R=\prod R_{i}$. However, the only central orthogonal idempotents of $R$ are the sums of the elements $1_{R_{i}}$ (the identity element of $R_{i}$ ). And it is clear from the definition of $T$ that the only such sums belonging to $T$ are 1 and 0 . Hence $T$ is indecomposable.

(ii) Evidently $R=\sum 1_{R_{i}} T$ and so is a finite centralizing extension of $T$. The result follows from [5] or [8].

(iii) Suppose $X$ is a nilpotent ideal of $T$. The isomorphism in Lemma 2.2(iv) shows, for each $j$, that $X \subseteq \prod_{i \neq j} A_{i}$; and so $X=0$. Clearly $T$ is not prime, since $A_{1} A_{2}=0$.

In order to apply the global dimension results from Section 1 , it is necessary to choose the rings $R_{i}$ with care. Note that 0 ( ) denotes an idealizer.

Notation 2.4. For $i=1, \ldots, n$, let $S_{i}$ be a ring, let $A_{i}$ be a proper right ideal of $S_{i}$ with $S_{i} A_{i}=S_{i}$ and let $R_{i}$ be a subring of $\mathbb{D}\left(A_{i}\right)$ containing $A_{i}$ and with $R_{i} / A_{i} \simeq K$, a semisimple ring. Let $T$ be as specified in 2.1. Let $S=\prod S_{i}, R=\prod R_{i}$ and $A=\prod A_{i}$.

Proposition 2.5.

(i) $\mathrm{rt} \operatorname{gl} \operatorname{dim} S \leqslant \mathrm{rt} \operatorname{gl} \operatorname{dim} T \leqslant 1+\mathrm{rt} \operatorname{gl} \operatorname{dim} S$.

(ii) If $\mathrm{rt} \mathrm{gl} \operatorname{dim} S=0$ then $\mathrm{rt} \mathrm{gl} \operatorname{dim} T=1$. 
(iii) p.d. $(T / A)_{T}=$ p.d. $(S / A)_{S}$.

(iv) If p.d. $(S / A)_{S}=0$ then p.d. $(S / R)_{T} \leqslant 1$.

(v) If p.d.(S/A $)_{S}>0$ then p.d. $(S / R)_{T}=1+$ p.d.(S/A $)_{S}$.

Proof.

(i) By construction $T / A \simeq K$ which is semisimple; and $S A=S$. So Corollary 1.4 applies.

(ii) This is clear from (i) together with Lemma 1.1(ii).

(iii) This follows from Lemma 1.1(iii).

(iv) Since p.d.(S/A $)_{S}=0$, then $A_{S}, A_{T}$ and each $\left(A_{i}\right)_{T}$ has an idempotent generator (as in the proof of Lemma 1.1(iii)). But $R_{i} \simeq T / \prod_{i \neq i} A_{i}$ and so p.d. $R_{T}=0$. Hence p.d. $(S / R)_{\mathrm{T}} \leqslant 1$.

(v) Since p.d.(S/A $)_{S}>0$, it follows that $A_{S}$, and hence $A_{T}$ also, does not have an idempotent generator. The same, therefore, is true of $\left(A_{k}\right)_{T}$ for at least one $k \in\{1, \ldots, n\}$. Hence the short exact sequences $0 \rightarrow A \rightarrow T \rightarrow T / A \rightarrow 0$ and $0 \rightarrow \prod_{j \neq i} A_{j} \rightarrow T \rightarrow R_{i} \rightarrow 0$ are nonsplit, provided $i \neq k$. Hence p.d. $(T / A)_{T}=1+$ p.d. $A_{T}$ and p.d. $\left(R_{i}\right)_{T}=$ $1+\sup _{j \neq i}$ p.d. $\left(A_{j}\right)_{T}$. Therefore

$$
\text { p.d. } R_{T}=\sup \left\{\text { p.d. }\left(R_{i}\right)_{T}\right\}=1+\sup \left\{\text { p.d. }\left(A_{\mathrm{j}}\right)_{\mathrm{T}}\right\}=1+\text { p.d. } A_{\mathrm{T}} \text {. }
$$

Since p.d. $R_{\mathrm{T}}>0$, then p.d. $(S / R)_{\mathrm{T}}=1+$ p.d. $R_{\mathrm{T}}$ and thus

using (iii).

$$
\text { p.d. }(S / R)_{T}=2+\text { p.d. } A_{T}=1+\text { p.d. }(T / A)_{T}=1+\text { p.d. }(S / A)_{S},
$$

With more restrictions on $R_{i}$ and $A_{i}$ more precision can be achieved, as the next two results show.

Theorem 2.6. If p.d. $(S / A)_{S}=\mathrm{rt} g l \operatorname{dim} S$, then $\mathrm{rt} g l \operatorname{dim} T=1+\mathrm{rt} g l \operatorname{dim} S$.

Proof. If p.d.(S/A) $)_{S}=0$ then $\mathrm{rt} g \mathrm{dim} S=0$ and the result follows from Proposition 2.5(ii). On the other hand, if p.d. $(S / A)_{S}>0$, then Proposition 2.5(v) shows that p.d. $(S / R)_{T}=1+$ p.d. $(S / A)_{S}=1+\mathrm{rt} g l \operatorname{dim} S$ and so, by Proposition $2.5(\mathrm{i})$, rt $\mathrm{gl} \operatorname{dim} T=$ $1+\mathrm{rt} \operatorname{gl} \operatorname{dim} S$.

THEOREM 2.7. If, for each $i, R_{i}=\llbracket\left(A_{i}\right)$ with $A_{i}$ being a semimaximal right ideal of $S_{i}$, then

$$
\mathrm{rt} \operatorname{gl} \operatorname{dim} T=\sup \left\{\mathrm{rt} \operatorname{gl} \operatorname{dim} S, 1+\text { p.d. }(S / A)_{S}\right\} .
$$

Proof. If $U$ is a simple $T$-subfactor of $S / T$ then $U$ is a subfactor of either $(S / R)_{T}$ or $(R / T)_{T}$, each of which is semisimple. Therefore

$$
\sup \{\text { p.d. } U\}=\sup \left\{\text { p.d. }(S / R)_{T}, \text { p.d. }(R / T)_{T}\right\} \text {. }
$$

Now $R / T$ is a $T / A$-module, and $T / A$ is semisimple. Hence p.d. $(R / T)_{T} \leqslant$ p.d. $(T / A)_{T}=$ p.d. $(S / A)_{S}$ by Proposition 2.5(iii). Also, by Proposition 2.5 (iv) and (vi), p.d.(S/R $)_{T} \leqslant$ $1+$ p.d. $(S / A)_{\text {S. Hence }}$ sup\{p.d. $\left.U\right\} \leqslant 1+$ p.d. $(S / A)_{S}$ and the result follows from Theorem 1.2 . 
We now turn to some specific examples.

EXAMPLES 2.8 .

(a) Let $k$ be a field, $D$ be the commutative polynomial ring $k\left[x_{1}, \ldots, x_{p}\right]$ and $M=\left(x_{1}, \ldots, x_{p}\right)$. Then $M$ is a maximal ideal with p.d. $M=p$. For $i=1, \ldots, n$, let $S_{i}=M_{2}(D), A_{i}=\left[\begin{array}{cc}M & M \\ D & D\end{array}\right]$ and $R_{i}=\emptyset\left(A_{i}\right)=\left[\begin{array}{cc}D & M \\ D & D\end{array}\right]$. By [14, Theorems 2.2, 2.5], together with symmetry, $R_{i}$ is Noetherian with Krull dimension $p$; and clearly $R_{i}$ is a prime ring. Therefore by Lemma $2.3 T$ is a Noetherian, indecomposable semiprime ring with Krull dimension $p$ on each side.

Now $g l \operatorname{dim} S_{i}=g l \operatorname{dim} D=p$, and, since $S_{i} / A_{i}$ corresponds, under Morita equivalence, with $D / M$, then p.d. $\left(S_{i} / A_{i}\right)=p$. Thus p.d. $S / A=p$ and so, by Theorem 2.7 and symmetry, $\operatorname{gl} \operatorname{dim} T=p+1$. Evidently $T$ is also a P.I. ring.

(b) Let $k$ be a field of characteristic zero, and, for $i=1, \ldots, n$, let $S_{i}$ be the first Weyl algebra over $k$. It is well known that $S_{i}$ is a Noetherian integral domain with $\mathrm{K} \operatorname{dim} S_{i}=$ gl $\operatorname{dim} S_{i}=1$. Take $A_{i}$ to be any nonzero right ideal of $S_{i}$, and let $R_{i}=k+A_{i}$. By [14, Theorem 7.4] $R_{i}$ is a Noetherian integral domain of Krull dimension 1. So $T$ is an indecomposable Noetherian semiprime ring with $\mathrm{K} \operatorname{dim} T=1$. By Theorem 2.6, $\mathrm{rt} \operatorname{gl} \operatorname{dim} T=2$ and, since $T$ is Noetherian, $\mathrm{lt} \mathrm{gl} \operatorname{dim} T=2$. Since each $R_{\mathrm{i}}$ is primitive, $T$ is semiprimitive.

(c) In both the previous examples $\operatorname{gl} \operatorname{dim} T>\operatorname{gl} \operatorname{dim} S$. This is not always the case, however. For example, take $D, M$ as in (a), with $p \geqslant 2$, and let $E=\left[\begin{array}{ll}D & M \\ D & D\end{array}\right]$ which, as noted in (a), has global dimension $p$. As a right $E$-module $\mu_{2}(D) / E \approx E / L$ where $L=\left[\begin{array}{ll}D & M \\ D & M\end{array}\right]$ is a maximal right ideal of $E$. Hence p.d. $E / L=1$.

Let $S_{i}=M_{2}(E), A_{i}=\left[\begin{array}{ll}L & L \\ E & E\end{array}\right]$ and $R_{i}=\emptyset\left(A_{i}\right)$. Arguing as in (a), it follows that $T$ is Noetherian, with Krull dimension $p$, and that $\operatorname{gl} \operatorname{dim} T=\operatorname{gldim} S=p$.

NOTE 2.9. (i) We note, in connection with [3], that in none of these examples is it the case that p.d. $U=\mathrm{gl} \operatorname{dim} T$ for all simple $T$-modules $U$. In fact $T / A$ is a simple $T$-module and p.d. $T / A<\operatorname{gl} \operatorname{dim} T$. For, by Proposition $2.5(\mathrm{iii})$, p.d. $(T / A)_{T}=$ p.d. $(S / A)_{S}>0$ and then

$$
\text { p.d. }(T / A)_{T}<1+\text { p.d. }(S / A)_{S}=\text { p.d. }(S / R)_{T} \leqslant \operatorname{gl} \operatorname{dim} T
$$

by Proposition $2.5(\mathrm{v})$.

(ii) Let $T$ be a Noetherian semiprime, but not prime, indecomposable ring, finite over its centre $C$, as in Examples 2.8(a) and (c). Then $C$ is indecomposable, Noetherian, by [5] or [8], but not an integral domain [15, 1.6.14]. Thus $\mathrm{gl} \operatorname{dim} C=\infty$.

(iii) In [19, Chapters 3,4$]$ commutative rings of global dimension 2 are constructed by methods akin to those above. However, these are not Noetherian. 
3. Some prime rings of finite global dimension. In this section we describe the constructions of some subrings $R$ of a Dedekind prime ring $S$ which turn out to be Noetherian prime rings of finite global dimension. The basic facts required concerning $S$ can be found in [6]. In the particular case when $S=\mathcal{M}_{n}(D)$ for a discrete valuation ring $D$, these examples are amongst those considered by Tarsy $[17,18]$ and $V$. A. Jategaonkar $[10,11,12]$, and by Fields [7]. When $S$ is a (noncommutative) principal ideal domain, Example 3.7 becomes one of the examples used by Stafford and Warfield [16].

EXAMPle 3.1. Let $S$ be a Dedekind prime ring with a nonzero maximal ideal, $P$ say. Then $S / P^{3} \simeq \mathcal{M}_{n}(D)$ where $D$ is an Artinian principal ideal ring, with radical $M$ say, such that $D / M$ is a division ring and $M^{3}=0$. Under the isomorphism, $P / P^{3}$ corresponds to $\mu_{n}(M)$ and $P^{2} / P^{3}$ to $\mu_{n}\left(M^{2}\right)$.

Let $R$ be the subring of $S$ containing $P^{2}$ and with

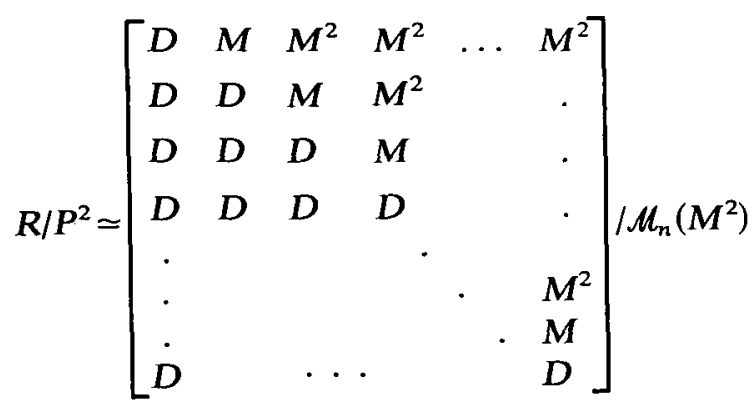

and let $\left\{e_{i j}\right\}$ be inverse images in $S$ of the matrix units of $\mu_{n}(D)$.

THEOREM 3.2. $R$ is a Noetherian prime ring with $\mathrm{K} \operatorname{dim} R=1$, and with $\operatorname{gl} \operatorname{dim} R=$ $n-1$, provided $n \geqslant 2$.

We will split the proof into a number of steps. Note, however, that $R$ contains the right ideal $A$ of $S$ defined by $A=e_{n n} S+P^{2}$, and $S A=S$. Thus the results of Section 1 are applicable. It is easy to see that $(S / A)_{R}$ has finite length-and indeed a composition series will be exhibited later. This, by Theorem 1.5 , shows that $R$ is right Noetherian and, by symmetry, left Noetherian and that $\mathrm{K} \operatorname{dim} R=1$. The fact that $P^{2} \subseteq R$ shows that $R$ and $S$ have the same quotient ring and so $R$ is also prime.

In order to obtain the global dimension, it is convenient to consider "one-rowed" right $R$-modules, i.e. right $R$-modules $B$ with $P^{2} \subseteq B \subseteq e_{i j} S+P^{2}$ for some $i$, $j$.

LemMa 3.3. (i) For any $i, j, e_{i j} R+P^{2}$ is projective.

(ii) If $B_{R} \subseteq e_{i i} S+P^{2}$ and $B_{R}^{\prime} \subseteq e_{i j} S+P^{2}$ satisfy $e_{j i} B+P^{2}=B^{\prime}, e_{i j} B^{\prime}+P^{2}=B$, then p.d. $B=$ p.d. $B^{\prime}$.

Proof. (i) The map $\left(\left(1-e_{i j}\right) R+P^{2}\right) \oplus\left(e_{i j} R+P^{2}\right) \rightarrow R$, given by addition, is clearly surjective and so splits; and it has kernel $P^{2}$. However, $P^{2}$ is a projective right ideal of $S$ and hence, by Lemma 1.1(i), of $R$. 
(ii) The map $\alpha: B \oplus P^{2} \rightarrow B^{\prime}$ via $(b, x) \rightarrow e_{i i} b+x$ yields a short exact sequence

$$
0 \rightarrow P^{2} \rightarrow B \oplus P^{2} \stackrel{\alpha}{\rightarrow} B^{\prime} \rightarrow 0
$$

and it is thus clear that p.d. $B=$ p.d. $B^{\prime}$.

Thus, in effect, each row is the same as any other. We can therefore concentrate on one row-the first say. We set $I=I\left(n_{1}, n_{2}, n_{3}\right)$ to be the $R$-submodule of $e_{11} S+P^{2}$ containing $P^{2}$ and with

$$
I / P^{2} \simeq\left[\begin{array}{lcr}
D \ldots D & M \ldots M & M^{2} \ldots M^{2} \\
0 & \ldots & 0 \\
0 & \ldots &
\end{array}\right]+\mathcal{M}_{n}\left(M^{2}\right) / M_{n}\left(M^{2}\right)
$$

where the numbers of $D, M$ and $M^{2}$ terms in the first row are $n_{1}, n_{2}, n_{3}$ respectively.

Similarly, $J=J\left(n_{1}, n_{2}, n_{3}\right)$ is the $R$-submodule of $e_{11} S+P^{3}$ containing $P^{3}$ and with

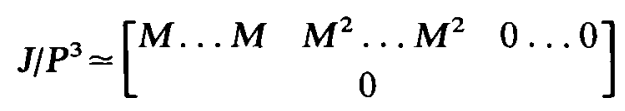

where $n_{1}, n_{2}, n_{3}$ are the numbers of $M, M^{2}$ and 0 terms in the first row.

LEMMA 3.4. (i) p.d. $J\left(n_{1}, n_{2}, n_{3}\right)=$ p.d. $I\left(n_{1}, n_{2}, n_{3}\right)$.

(ii) If $n_{1}>0$ then $I\left(n_{1}, 1, n-n_{1}-1\right)$ is projective.

(iii) If $n_{1}>0$ and $n_{2}>1$ then $I\left(n_{1}, n_{2}, n_{3}\right)$ is not projective.

(iv) If $n_{1}>0$ and $n_{2} \geqslant 1$ then p.d. $I\left(n_{1}, n_{2}, n_{3}\right)=n_{2}-1$.

Proof. (i) Note that both $P$ and $P^{-1}$ are projective as right or left $R$-modules, and that $I \otimes P \simeq J, J \otimes P^{-1} \simeq I$. Thus any projective resolution for $I$ yields one for $J$ and vice versa.

(ii) This follows immediately from Lemma 3.3.

(iii) We may view $I^{*}$, the dual of $I$, as a subset of $Q$, the quotient ring of $R$ and of $S$. Evidently $I^{*} / P^{2} \subseteq P^{-2} / P^{2}$ which can again be viewed as a collection of $n \times n$ matrices $\left(\simeq S / P^{4}\right.$ in fact $)$. It is easy to check that the matrices in $I^{*} / P^{2}$ must have elements only from $M$ in the first $n_{1}$ entries of column 1 . But that alone ensures that $1 \notin I I^{*}$ and so, by the dual basis lemma, $I$ is not projective.

(iv) Note, by (ii) and (iii), if $n_{1}>0$, then $I\left(n_{1}, n_{2}, n_{3}\right)$ is projective if and only if $n_{2}=1$. It is easy to check that

and

$$
I\left(n_{1}, n_{2}, n_{3}\right)=I\left(n_{1}, 1, n_{2}+n_{3}-1\right)+J\left(n_{1}+n_{2}, 1, n_{3}-1\right)
$$

$$
J\left(n_{1}+1, n_{2}-1, n_{3}\right)=I\left(n_{1}, 1, n_{2}+n_{3}-1\right) \cap J\left(n_{1}+n_{2}, 1, n_{3}-1\right) .
$$

This, of course, yields a short exact sequence whose middle term is projective by (i) and (ii). By (i) the kernel has the same projective dimension as has $I\left(n_{1}+1, n_{2}-1, n_{3}\right)$. Induction on $n_{2}$ yields the result. 
Proof of Theorem 3.2. The chain

$$
I(n, 0,0) \supset I(n-1,1,0) \supset \ldots \supset I(1, n-1,0) \supset I(1, n-2,1) \supset \ldots \supset I(1,1, n-2)
$$

together with the similar chains inside each $e_{i i} S+P^{2}, i \geqslant 2$, has simple factors and includes all the simple modules $U$ required in order to apply Theorem 1.2. It is clear from 3.4 that sup\{p.d. $U\}=n-1$, with the supremum being achieved by $U=I(2, n-2,0) / I(1, n-1,0)$. Hence $\mathrm{gl} \operatorname{dim} R=n-1$.

Note 3.5. Arguments similar to those involved in Theorem 3.2 apply if $P$ is a finite intersection of nonzero maximal ideals of $S$. Then $S / P^{3}$ is a direct product of matrix rings as described in Example 3.1, $\Pi \mathcal{M}_{n_{i}}\left(D_{i}\right)$ say. If $R$ is chosen to be the subring containing $P^{2}$, with $R / P^{2}$ being the direct product of the appropriate subrings of these matrix rings then $\operatorname{gl} \operatorname{dim} R=\sup \left\{n_{i}-1\right\}$ provided some $n_{i} \geqslant 2$. Again $R$ is prime Noetherian of Krull dimension 1 .

COROLlaRY 3.6 [10]. If $B$ is a (commutative) discrete valuation ring with maximal ideal $M$ then the subring

$$
\left[\begin{array}{ccccc}
B & M & M^{2} & \ldots & M^{2} \\
B & B & M & & \cdot \\
\cdot & & & & \cdot \\
\cdot & & & & \cdot \\
\cdot & & & & M^{2} \\
& & & & M \\
B & B & B & \ldots & B
\end{array}\right]
$$

of $\mathcal{M}_{n}(B)$ has global dimension $n-1$.

ExAmple 3.7. First, let $K$ be a division ring. We aim to describe a subring $T_{n}$ of $\mu_{2^{n}}(K)$. Let $T_{1}=\left[\begin{array}{cc}K & 0 \\ K & K\end{array}\right] \subseteq M_{2}(K)$, and, inductively, $T_{m+1}=\left[\begin{array}{cc}T_{m} & 0 \\ T_{m} & T_{m}\end{array}\right] \subseteq \mu_{2}\left(T_{m}\right)$.

Now let $S$ be a Dedekind prime ring, $P$ a maximal ideal such that $S / P \simeq \mathscr{M}_{2^{n}}(K)$, and let $R$ be the inverse image in $S$ of $T_{n}$.

THEOREM 3.8. $R$ is a Noetherian prime ring of Krull dimension 1 and global dimen$\operatorname{sion} n$.

The proof is similar to that of Theorem 3.2 although some of the details are rather more complicated. We merely sketch the proof.

Note first that the right ideal $A$ of $S$ which is the inverse image in $S$ of the right ideal

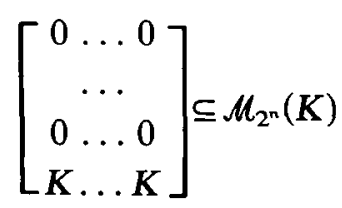


is, in fact, a right ideal of $R$ and, moreover, $S A=S$. Thus the results of Section 1 are applicable. Furthermore, $S / A$ has finite length over $K$ and hence over $R$. As before, it follows that $R$ is Noetherian of Krull dimension 1; and since $P \subseteq R, R$ is a prime ring with the same quotient ring as $S$.

In order to calculate the projective dimensions of simple composition factors of $S / R$ we consider a chain of submodules between the inverse images in $S$ of $e_{11} \mu_{2^{n}}(K)$ and $e_{11} T_{n}$, i.e. of

$$
\left[\begin{array}{c}
K K \ldots K \\
0
\end{array}\right] \text { and }\left[\begin{array}{c}
K 0 \ldots 0 \\
0
\end{array}\right]
$$

First we describe the chain inside $\mathcal{M}_{2^{n}}(K)$. Set $A_{0}, A_{1}, A_{2}, \ldots$ to be

$$
\left[\begin{array}{c}
K K \ldots K K K \\
0
\end{array}\right],\left[\begin{array}{c}
K K \ldots K K \\
0
\end{array}\right],\left[\begin{array}{c}
K K \ldots K \\
0
\end{array}\right], \ldots
$$

or

$$
[K K \ldots K K K],[K K \ldots K K 0],[K K \ldots K 00], \ldots
$$

for short. Let $A_{0}^{*}, A_{1}^{*}, A_{2}^{*}, \ldots$ be their inverse images in $S$. These are the "one-rowed" right $R$-modules of interest here; and again it is enough to concentrate just on the first row. Note that the only $A_{i}^{*}$ which are projective are those with $A_{i}$ being identical with one of the rows of $T_{n}$. Such $A_{i}$ we will call principal projectives of $T_{n}$. Now each $A_{i}$ can be subdivided, taking the first and second halves of its entries, to give two similar rows in $T_{n-1}$ : say $A_{i}=\left(B_{i}, C_{i}\right)$. Inductively, we suppose projective resolutions of $B_{i}$ and $C_{i}$ are known, involving only principal projectives of $T_{n-1}$, and maps involving additions of rows. Thus if $D$ is a one-rowed module from $T_{n-1}$ let

$$
0 \rightarrow Q_{r} \rightarrow \ldots \rightarrow Q_{2} \rightarrow Q_{1} \rightarrow D \rightarrow 0
$$

be the projective resolution for $D$ with each $Q_{j}=\sum \oplus P_{j k}$ where the $P_{i k}$ are principal projectives of $T_{n-1}$.

Now, given any principal projective $P$ of $T_{n-1}$, there are two related principal projectives of $T_{n}$ : one formed by preceding $P$ by $K^{\left(2^{n-1}\right)}$, the other by following $P$ by $2^{n-1}$ zeros. We write $(\mathbf{K}, P)$ and $(P, \mathbf{0})$ for short, and let $\left(\mathbf{K}, Q_{i}\right)$ denote $\sum \oplus\left(\mathbf{K}, P_{j k}\right)$. Then

$$
\begin{aligned}
0 \rightarrow\left(Q_{r}, \mathbf{0}\right) \rightarrow\left(Q_{r-1}, \mathbf{0}\right) \oplus\left(Q_{r}, Q_{r}\right) & \rightarrow \ldots \rightarrow \\
& \left(Q_{1}, \mathbf{0}\right) \oplus\left(Q_{2}, Q_{2}\right) \rightarrow(\mathbf{K}, \mathbf{0}) \oplus\left(Q_{1}, Q_{1}\right) \rightarrow(\mathbf{K}, D) \rightarrow 0
\end{aligned}
$$

and

$$
0 \rightarrow\left(Q_{r}, \mathbf{0}\right) \rightarrow \ldots \rightarrow\left(Q_{2}, \mathbf{0}\right) \rightarrow\left(Q_{1}, \mathbf{0}\right) \rightarrow(D, \mathbf{0}) \rightarrow 0
$$

are both projective resolutions of these one-rowed modules of $T_{n}$ of the same type.

Note next that each $A_{i}$ either has the form $(\mathbf{K}, D)$ or $(D, \mathbf{0})$ for some $D$. Thus we obtain projective resolutions for each $A_{i}$, of maximal length $n-1$. The form of these resolutions means that they can easily be lifted to give resolutions of the $A_{i}^{*}$; and, as before, it can be checked that these are indeed of minimal length. The result then follows from 1.2. 


\section{REFERENCES}

1. K. A. Brown, C. R. Hajarnavis and A. B. MacEacharn, Noetherian rings of finite global dimension, Proc. London Math. Soc. 44 (1982), 349-371.

2. K. A. Brown, C. R. Hajarnavis and A. B. MacEacharn, Rings of finite global dimension integral over their centres, Comm. Algebra, 11 (1983), 67-93.

3. K. A. Brown and C. R. Hajarnavis, Homologically homogeneous rings, Trans. Amer. Math. Soc. 281 (1984), 197-208.

4. A. W. Chatters and C. R. Hajarnavis, Rings with Chain Conditions (Pitman, 1980).

5. D. Eisenbud, Subrings of Artinian and Noetherian rings, Math. Ann. 185 (1970), 247-249. 67-85.

6. D. Eisenbud and J. C. Robson, Modules over Dedekind prime rings, J. Algebra 16 (1970), $126-130$

7. K. L. Fields, Examples of orders over discrete valuation rings, Math. Z. 111 (1969),

8. E. Formanek and A. V. Jategaonkar, Subrings of Noetherian rings, Proc. Amer. Math. Soc. 46 (1974), 181-186.

9. K. R. Goodearl, Subrings of idealizer rings, J. Algebra 33 (1975), 405-429.

10. V. A. Jategaonkar, Global dimension of triangular orders over a discrete valuation ring, Proc. Amer. Math. Soc. 38 (1973), 8-14.

11. V. A. Jategaonkar, Global dimension of tiled orders over commutative Noetherian domains, Trans. Amer. Math. Soc. 190 (1974), 357-374.

12. V. A. Jategaonkar, Global dimension of tiled orders over a discrete valuation ring, Trans. Amer. Math. Soc. 196 (1974), 313-330.

13. L. S. Levy, Torsionfree and divisible modules over non-integral domains, Canad. J. Math. 15 (1963), 132-151. 45-81.

14. J. C. Robson, Idealizers and hereditary Noetherian prime rings, J. Algebra 22 (1972),

15. L. H. Rowen, Polynomial Identities in Ring Theory (Academic Press, 1980).

16. J. T. Stafford and R. B. Warfield, Constructions of hereditary Noetherian rings and simple rings, Proc. London Math. Soc., to appear.

17. R. B. Tarsy, Global dimension of orders, Trans. Amer. Math. Soc. 151 (1970), 335-340. 423-426.

18. R. B. Tarsy, Global dimension of triangular orders, Proc. Amer. Math. Soc. 28 (1971),

19. W. V. Vasconcelos, The Rings of Dimension Two (Dekker, 1976).

SCHOOL of Mathematics

UNIVERSITY OF LEEDS

LEEDS

ENGLAND

LS2 9JT 